\title{
Mitophagy contributes to endothelial adaptation to simulated microgravity
}

\author{
Laura Locatelli $^{1}$ ｜ Alessandra Cazzaniga ${ }^{1}$ | Clara De Palma ${ }^{2}$ | Sara Castiglioni ${ }^{1}$ | \\ Jeanette A. M. Maier ${ }^{1}$
}

${ }^{1}$ Department Biomedical and Clinical Sciences L. Sacco, Università di Milano, Milano, Italy

${ }^{2}$ Unit of Clinical Pharmacology, Luigi Sacco University Hospital, ASST

Fatebenefratelli Sacco, Milano, Italy

\section{Correspondence}

Jeanette A. M. Maier, Department Biomedical and Clinical Sciences L. Sacco, Università di Milano, Via GB Grassi 74, 20157, Milano, Italy.

Email: jeanette.maier@unimi.it

Funding information

Agenzia Spaziale Italiana (ASI), Grant/ Award Number: ENDOSTEO Project

\begin{abstract}
Exposure to real or simulated microgravity is sensed as a stress by mammalian cells, which activate a complex adaptive response. In human primary endothelial cells, we have recently shown the sequential intervention of various stress proteins which are crucial to prevent apoptosis and maintain cell function. We here demonstrate that mitophagy contributes to endothelial adaptation to gravitational unloading. After 4 and $10 \mathrm{~d}$ of exposure to simulated microgravity in the rotating wall vessel, the amount of BCL2 interacting protein 3, a marker of mitophagy, is increased and, in parallel, mitochondrial content, oxygen consumption, and maximal respiratory capacity are reduced, suggesting the acquisition of a thrifty phenotype to meet the novel metabolic challenges generated by gravitational unloading. Moreover, we suggest that microgravity induced-disorganization of the actin cytoskeleton triggers mitophagy, thus creating a connection between cytoskeletal dynamics and mitochondrial content upon gravitational unloading.
\end{abstract}

\section{K E Y W O R D S}

cytoskeleton, HUVEC, mitochondria, RWV

\section{1 | INTRODUCTION}

Microgravity affects health and this results from an array of adaptive responses that ultimately influence homeostasis. ${ }^{1,2}$ It is of particular relevance that microgravity alters the behavior of endothelial cells (EC), ${ }^{3}$ the gatekeepers of vascular integrity and function, crucial to provide continued and adequate perfusion to all the tissues to sustain their own needs. ${ }^{4}$ EC react to many different stimuli. Being located at the interface between the blood and the tissues, these cells are susceptible to the action of a large variety of soluble factors. Moreover, they sense mechanical forces, such as shear stress, a frictional force acting in the direction of blood flow, pressure-stretch, which acts perpendicularly to the vascular wall, ${ }^{5}$ and gravity. ${ }^{6}$ Indeed, many studies have demonstrated that both real and simulated microgravity affect the behavior of human umbilical vein endothelial cell (HUVEC), ${ }^{7-14}$ a consolidated model of macrovascular EC. Microgravity models the organization of the cytoskeleton, the proliferative rate, the production of inflammatory, and vasoactive molecules, ${ }^{7-14}$ partly through the activation of a dynamic adaptive response which prevents apoptosis and generates a novel balance so that essential cell functions are preserved. ${ }^{15}$ In face of all this body of knowledge, no data are available on HUVEC bioenergetics in microgravity. In physiological conditions, EC obtain most of the energy by converting glucose to lactate, ${ }^{16}$ with two principal

Abbreviations: BNIP3, BCL2 interacting protein 3; CQ, chloroquine; CTR, 1g condition control; CYP D, cyclophilin D; EC, endothelial cells; FBS, fetal bovine serum; GAPDH, glyceraldehydes-3-phosphate dehydrogenase; HUVEC, human umbilical vein endothelial cells; ISS, international space station; LC3 B, microtubule-associated proteins 1A/1B light chain 3B; MTCO1, mitochondrially encoded cytochrome C oxidase I; NAC, N-acetyl-cysteine; P62, sequestosome 1; ROS, reactive oxygen species; RWV, rotating wall vessel; VDAC, voltage-dependent anion channels. 
goals, (a) to protect themselves from oxidative stress and (b) to preserve oxygen for the diffusion into the perivascular tissues. ${ }^{16,17}$ Therefore, in EC mitochondria, rod-shaped organelles that convert oxygen and nutrients into ATP, mainly serve as a biosynthetic center to produce the precursors necessary for the synthesis of various molecules required for cellular necessities. ${ }^{18}$ Moreover, mitochondria integrate signals from the environment, perceive cellular stresses, are vital in cell death signaling and many other functions, thus playing a crucial role in orchestrating cell behavior. ${ }^{19}$ Different stimuli are able to modulate mitochondrial content and function. Mitochondrial content is determined by the balance between mitochondrial biogenesis and degradation through the process called mitophagy, ie, the autophagic removal of damaged or superfluous mitochondria, while their organization, fundamental in determining their function, is the result of the balance between fusion and fission. ${ }^{20}$ Mitochondrial dynamics is strictly linked to cytoskeletal organization, which has a relevant role in maintaining mitochondrial network and function. $^{21}$

Because research in space is limited by the high costs and low flight opportunities, several ground-based tools have been developed for simulating microgravity on Earth, ${ }^{22}$ all based on eliminating a preferential direction of the gravity vector by continuous rotation. It is interesting to point that experiments performed in different bioreactors yielded the same results and often validate data obtained in space. ${ }^{10,15,22,23}$ We have utilized the NASA-developed rotating wall vessel (RWV), which generates a vector-averaged gravity similar to that of near-Earth free-fall orbit. ${ }^{3}$ In a previous study, we have shown the complex and sequential intervention of proteins involved in endothelial adaptive response after 4 and $10 \mathrm{~d}$ in the RWV. ${ }^{15}$ Here, we utilize the same time points and demonstrate that simulated microgravity activates mitophagy, thus reducing mitochondrial content and oxygen consumption in HUVEC.

\section{2 | MATERIALS AND METHODS}

\subsection{Cell culture}

HUVEC were from ATCC and serially passaged in M199 containing $10 \%$ fetal bovine serum, Endothelial Cell Growth Factor $(150 \mu \mathrm{g} / \mathrm{mL})$, glutamine $(2 \mathrm{mM})$, sodium pyruvate $(1 \mathrm{mM})$, and heparin $(5 \mathrm{U} / \mathrm{mL})$ on $2 \%$ gelatin coated dishes. All culture reagents were from Gibco (Thermo Fisher Scientific, Waltham, MA, USA). To generate microgravity, we utilized the RWV (Synthecon Inc, Houston, TX, USA) after seeding EC on beads (Cytodex 3, Sigma Aldrich, St Louis, MO, USA). ${ }^{7,10,24}$ As $1 \mathrm{~g}$ condition control (CTR), HUVEC grown on beads were cultured in the vessels not undergoing rotation. In some experiments, to allow the accumulation of autophagosomes, HUVEC were treated with $40 \mu \mathrm{M}$ of chloroquine (CQ) (Sigma Aldrich, St Louis, MO, USA) for 1 hour and then collected for protein extraction and western blot. To inhibit autophagy/mitophagy, ${ }^{25}$ HUVEC in the RWV were treated with $40 \mu \mathrm{M}$ of $\mathrm{CQ}$ for $4 \mathrm{~d}$ and collected for western blot and immunofluorescence. To contrast the formation of reactive oxygen species (ROS), we used N-acetyl-cysteine (NAC, Sigma Aldrich, St Louis, MO, USA) ${ }^{26}$ (1 mM) for 4 hours, since we detected higher amounts of ROS after 2 hours culture in the RWV. ${ }^{15}$ Therefore, some samples were removed from the RWV 2 and 4 hours after the beginning of the experiment to measure the levels of ROS using $2{ }^{\prime}-7^{\prime}$-dichlorofluorescein diacetate (DCFH) as described, ${ }^{15}$ while the others were maintained in the RWV for $10 \mathrm{~d}$ to evaluate mitochondrial mass.

In other experiments, HUVEC in $1 \mathrm{~g}$ condition were treated with $0.5 \mu \mathrm{M}$ of Cytochalasin D (Sigma Aldrich, St Louis, MO, USA).

\subsection{Oxygen consumption measurements}

The respiratory chain capacity was determined by high-resolution respirometry on HUVEC grown in $1 \mathrm{~g}$ condition or in the RWV for 4 and $10 \mathrm{~d}$. The beads were collected from the RWV vessels, trypsinized and $1 \times 10^{6}$ cells for each sample were used. The mitochondria respiration rates were measured using the $\mathrm{O} 2 \mathrm{~K}$ oxygraph chambers (Oroboros, Instruments, Innsbruck, Austria). The cells were transferred into oxygraph chambers and resuspended in the respiration medium MiR06 (0.5 mM EGTA, $3 \mathrm{mM} \mathrm{MgCl}_{2}$,

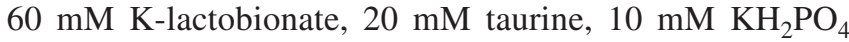
$20 \mathrm{mM}$ Hepes, $110 \mathrm{mM}$ sucrose, and $1 \mathrm{~g} / \mathrm{L}$ bovine serum albumin fatty acid-free, $280 \mathrm{U} / \mathrm{mL}$ catalase $(\mathrm{pH} 7.1))$. The sequential addition of pyruvate $(10 \mathrm{mM})$, malate $(2 \mathrm{mM})$, oligomycin $(0.5 \mu \mathrm{M})$, the uncoupler FCCP (Carbonyl cyanide-4-(trifluoromethoxy) phenylhydrazone, $0.5 \mu \mathrm{M}$ ), rotenone $(0.5 \mu \mathrm{M})$, and antimycin A (AA) $(2.5 \mu \mathrm{M})$ allows the measurement of basal, leak, and maximal respiration, respectively. All the reagents used for respirometry assay were purchased from Sigma.

Oxygen consumption was measured also on extracted mitochondria of HUVEC grown in $1 \mathrm{~g}$ condition or in the RWV for $4 \mathrm{~d}$. The cells were trypsinized, permeabilized, resuspended in isolation buffer $(100 \mathrm{mM} \mathrm{KCl}, 50 \mathrm{mM}$ TRIS, $5 \mathrm{mM} \mathrm{MgCl} 2,1.8 \mathrm{mM}$ ATP, $1 \mathrm{mM}$ EDTA), and centrifuged at $600 \mathrm{~g}$ for 10 minutes at $4^{\circ} \mathrm{C}$. Then, the supernatant was centrifuged at $10000 \mathrm{~g}$ for 15 minutes at $4^{\circ} \mathrm{C}$ to allow the sedimentation of the mitochondria which were resuspended in Mito Preservation Medium (MiR06 supplemented with $20 \mathrm{mM}$ Histidine, $20 \mu \mathrm{M}$ Vitamin E succinate, $3 \mathrm{mM}$ Glutathione, $1 \mu \mathrm{M}$ Leupeptin, $2 \mathrm{mM}$ Glutamate, $2 \mathrm{mM}$ 
Malate, $2 \mathrm{mM} \mathrm{Mg-ATP),} \mathrm{and} 150 \mu \mathrm{g}$ of mitochondria were used for high-resolution respirometry analysis. The efficiency of the different components of the respiratory chain was measured: complex I respiration (state2), maximal oxidative phosphorylation capacity of complex I (state3), complexes I and II (state3+Succ), complex II function (state3+Suc$\mathrm{c}+$ Rot), and proton leak across the inner mitochondrial membrane (state4). Then, respiratory chain was inhibited by antimycin $\mathrm{A}(2.5 \mu \mathrm{M})$ to obtain the residual oxygen flux that was subtracted to each steady state to correct oxygen fluxes. The respiratory control ratio was calculated as the ratio between state 3 and state 4 showing a general measure of mitochondrial function. The oxygen consumption measurements were performed three times and the results are shown as the mean \pm standard deviation.

\section{3 | Western blot}

HUVEC were lysed in $10 \mathrm{mM}$ Tris- $\mathrm{HCl}(\mathrm{pH}$ 7.4) containing $3 \mathrm{mM} \mathrm{MgCl}_{2}, 10 \mathrm{mM} \mathrm{NaCl}, 0.1 \%$ SDS, $0.1 \%$ Triton X-100, $0.5 \mathrm{mM}$ EDTA, and protein inhibitors, separated on SDSPAGE and transferred to nitrocellulose sheets at $400 \mathrm{~mA}$ for 2 hours at $4^{\circ} \mathrm{C}$. Western analysis was performed using antibodies against LC3 B (Cell Signaling, Euroclone, Pero, Italy), p62, and Cyclophilin D (CYP D, Invitrogen, Carlsbad, CA, USA), MTCO1, and VDAC (Abcam, Cambridge, UK), BCL2 interacting protein 3 (BNIP3) (Sigma Aldrich, St Louis, MO, USA), and glyceraldehydes-3-phosphate dehydrogenase (Tebu Bio-Santa Cruz, Magenta, Italy). After extensive washing, secondary antibodies labeled with horseradish peroxidase (Amersham Pharmacia Biotech Italia, Cologno Monzese, Italy) were used. Immunoreactive proteins were detected by the SuperSignal chemiluminescence kit (Pierce, Life Technologies- ThermoFisher Scientific, Waltham, MA, USA). ${ }^{27}$ All the experiments were performed at least three times and a representative blot is shown. Images were analyzed using ImageJ.

\subsection{Confocal imaging}

After culture in the RWV, HUVEC were trypsinized and cytospun on Frosted microscope glasses, fixed in phosphatebuffered saline containing $4 \%$ paraformaldehyde and $2 \%$ sucrose $\mathrm{pH} 7.6$, permeabilized with Triton $0.3 \%$, incubated with anti-CYP D or anti-LC3 immunopurified IgGs overnight at $4^{\circ} \mathrm{C}$, and stained with an Alexa Fluor 488 and 546 secondary antibody (ThermoFisher Scientific, Waltham, MA, USA), respectively. In the experiments with Cytochalasin D, the cells were directly cultured on a coverslip and processed as described above. We used rhodamine-labeled phalloidin to visualize the cytoskeleton in Cytochalasin D-treated cells. For HUVEC on the beads, we utilized fluorescein isothiocyanate-labeled phalloidin (Sigma), because the beads autofluoresce in the red spectrum. 4',6-Diamidine-2'-phenylindole dihydrochloride (DAPI, Sigma) was used to stain the nuclei. Finally, cells were mounted with ProLong Gold Antifade Mountant (Invitrogen, Carlsbad, CA, USA) and images were acquired using a $40 \mathrm{X}$ objective in oil by a SP8 Leica confocal microscope.

\section{5 | Statistical analysis}

Statistical significance was determined using the Student's $t$ test and set at $P$ values less than .05 . In the figures $* P<.05$; $* * P<.01 ; * * * P<.001$.

\section{3 | RESULTS}

\subsection{Mitochondrial content in HUVEC exposed to simulated microgravity}

To have an overview of the mitochondrial content, we performed immunofluorescence on HUVEC cultured in the RWV or in $1 \mathrm{~g}$ condition. After 4 and $10 \mathrm{~d}$, the cells were trypsinized and cytospun on a glass coverslip. To label the mitochondria, we used an antibody against CYP D, a protein that is part of the permeability transition pore in the inner membrane of the mitochondria. Images were then acquired using a confocal microscope. Figure $1 \mathrm{~A}$ shows that HUVEC grown in the RWV have fewer mitochondria than their respective controls (CTR).

To confirm these results at the biochemical level, we investigated the total amounts of CYP D, Mitochondrially Encoded Cytochrome C Oxidase I (MTCO1), a protein encoded by mitochondrial DNA, and voltage-dependent anion channels (VDAC), located on the outer mitochondrial membrane. ${ }^{28}$ MTCO, VDAC, and CYP D are all significantly downregulated in HUVEC cultured in the RWV for 4 and $10 \mathrm{~d}$ (Figure 1B).

We asked whether microgravity-induced reduction of mitochondrial mass was reversible upon return to $1 \mathrm{~g}$ culture conditions. To this purpose, we cultured HUVEC in the RWV. Some samples were removed from the RWV after $7 \mathrm{~d}$ and maintained in vessels non-undergoing rotation for three more days to be compared with cells that remained in the RWV for the full duration of the experiment (10 d). Upon release from gravitational unloading, mitochondrial content increased as demonstrated by immunofluorescence (Figure 2A) and western blot (Figure 2B) using antibodies against CYP D. 
(A) ounval Zoom $4 x$
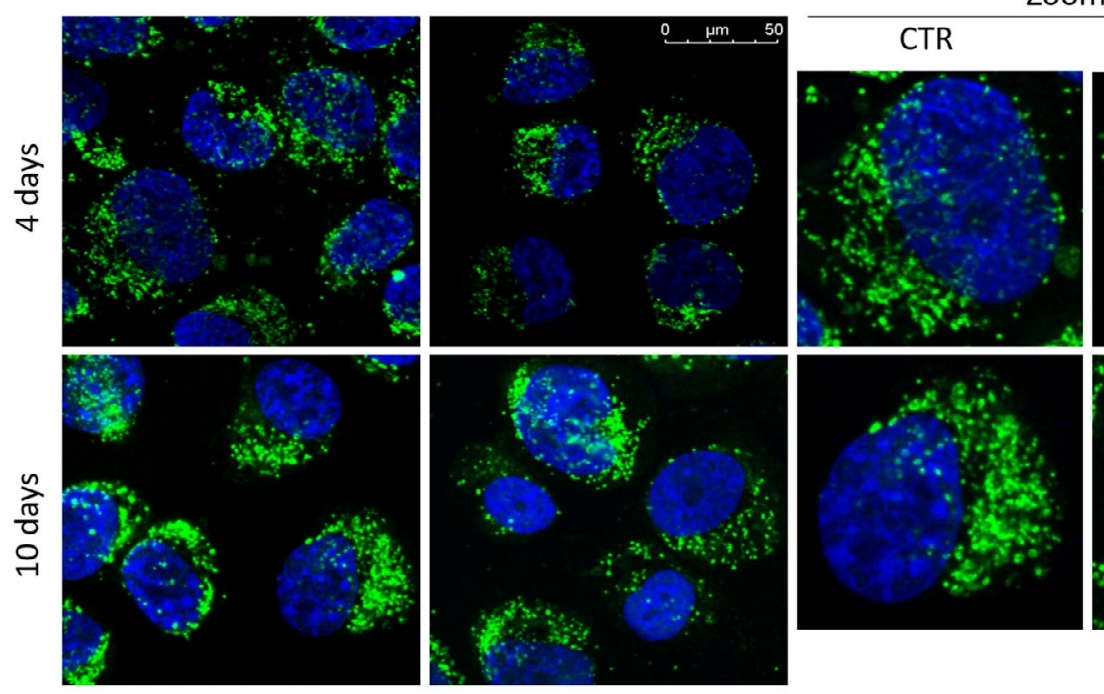

Zoom 11x
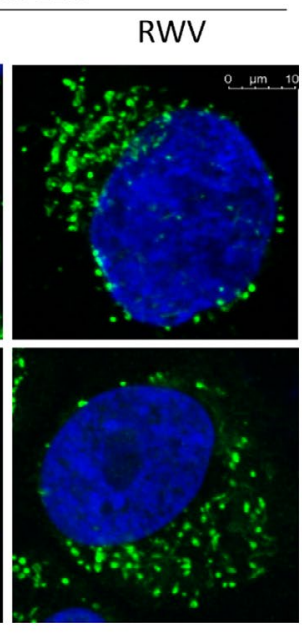

(B)
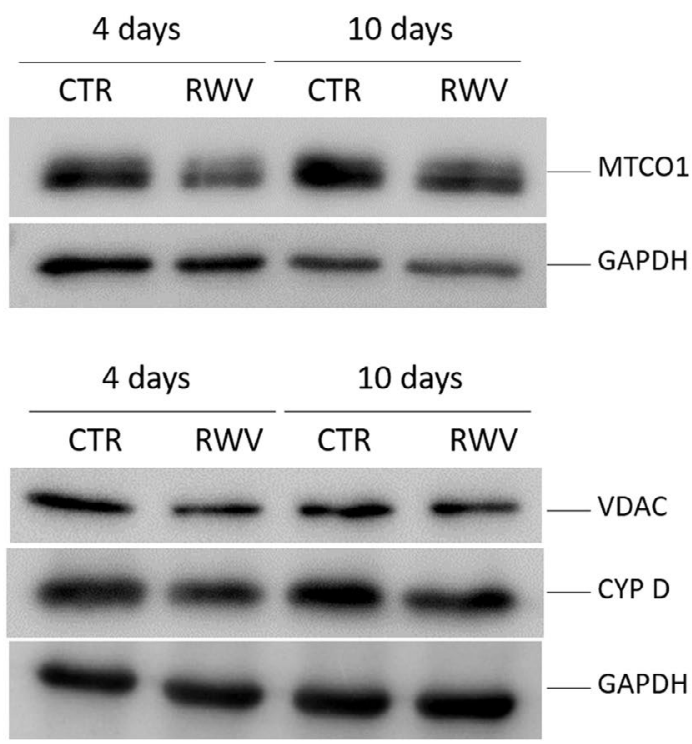
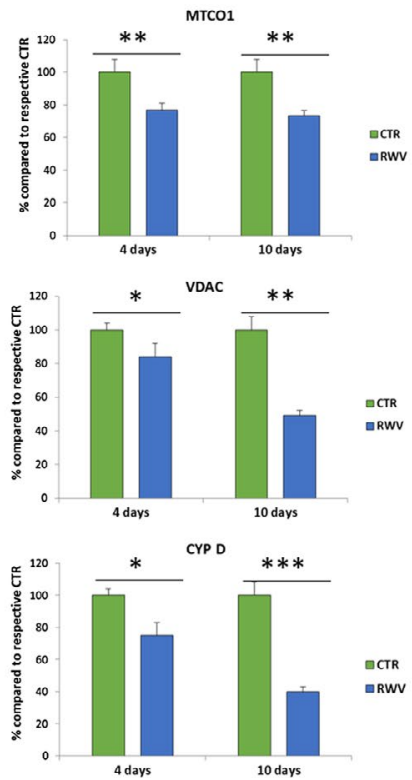

FI G URE 1 Visualization and quantification of mitochondria in HUVEC in simulated microgravity. HUVEC were grown in RWV or in $1 \mathrm{~g}$ condition (CTR) for 4 and $10 \mathrm{~d}$. A, After trypsinization, the cells were cytospun on glass coverslips and stained with DAPI to label the nuclei and CYP D to visualize the mitochondria. Images were acquired using a 40× objective in oil by a SP8 Leica confocal microscope. B, Western blots for different mitochondrial markers were performed. GAPDH was used as a control of loading. Representative blots (left) and densitometry obtained by ImageJ (right) are shown

\section{2 | Mitochondrial function in HUVEC exposed to simulated microgravity}

We investigated mitochondrial function in HUVEC using O2K oxygraph chambers. To this purpose, $1 \times 10^{6}$ cells for each sample were treated with different molecules to assess the efficiency of the different components of the respiratory chain. Different respiration states were measured. Maximal respiration represents the maximal activity of the electron transport chain, while basal respiration corresponds to oxygen consumption at steady state. Leak respiration measures oxygen consumption not linked to ATP production. HUVEC exposed to simulated microgravity tend to have a minor basal and leak capacity, without reaching statistical significance, compared to their controls, while their maximal respiratory capacity is significantly reduced (Figure 3A).

We also evaluated the function of isolated mitochondria in HUVEC in $1 \mathrm{~g}$ condition or exposed to simulated microgravity (RWV) for $4 \mathrm{~d}$. The respiratory control ratio, a 

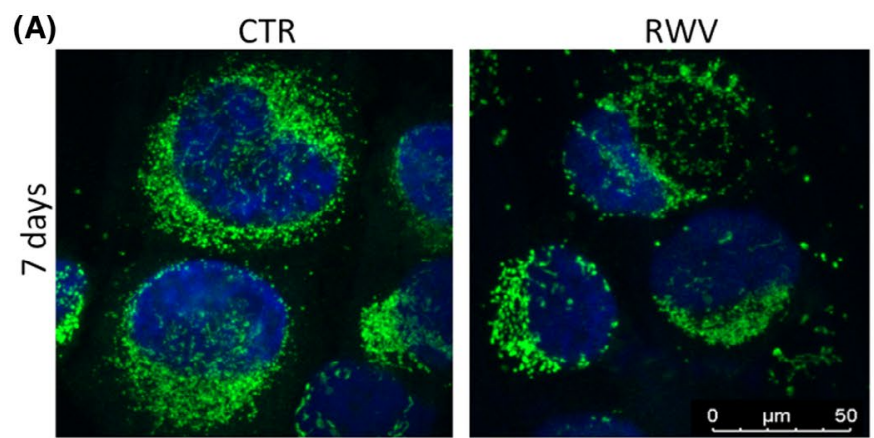

7 days RWV + 3days in $1 \mathrm{~g}$
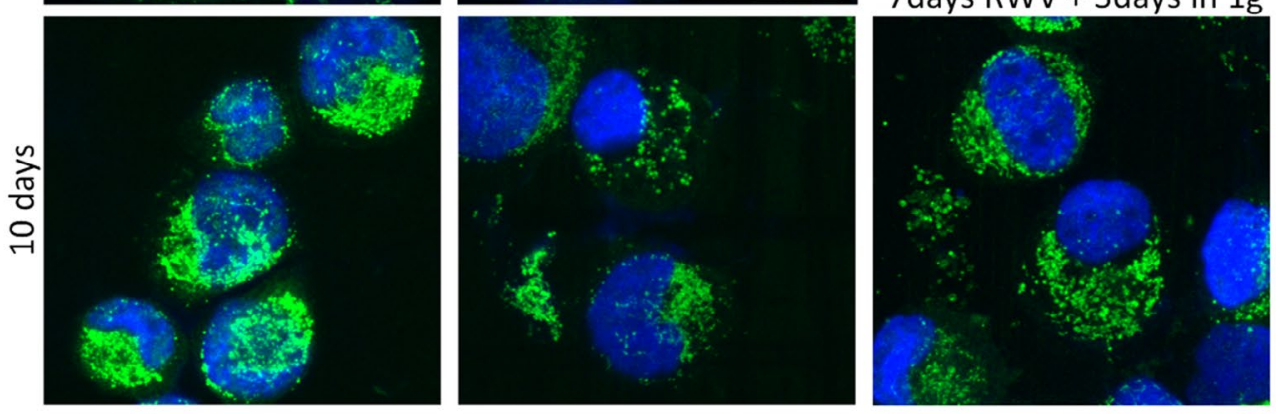

(B)
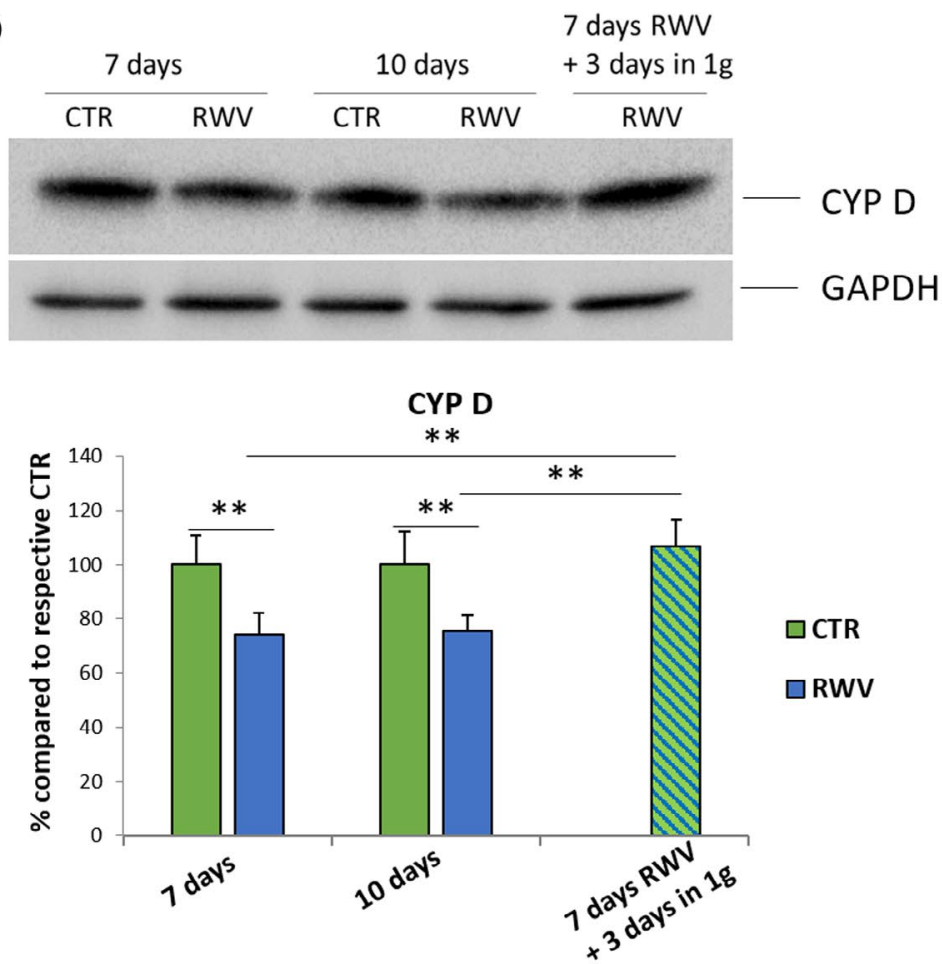

F I G URE 2 Visualization and quantification of mitochondria in HUVEC after return to $1 \mathrm{~g}$ culture conditions. HUVEC were cultured in the RWV for different times. After $7 \mathrm{~d}$, some samples were removed from the RWV and returned to $1 \mathrm{~g}$ for $3 \mathrm{~d}$, while the remaining samples were maintained in the RWV until the end of the experiment (day 10). A, Mitochondria were visualized utilizing antibodies against CYP D by immunofluorescence. B, Western blot was performed on cell lysates. A representative blot is shown (upper panel). Densitometry was performed using Image $\mathbf{J}$ (lower panel)

general measurement of mitochondrial function, was similar in controls and in cells in the RWV when the same amounts of mitochondria were used, indicating that mitochondria are as efficient in cells cultured in the RWV as in $1 \mathrm{~g}$ condition (Figure 3B).

\section{3 | Autophagy/mitophagy in HUVEC exposed to simulated microgravity}

We hypothesized that the decreased number of mitochondria in HUVEC exposed to simulated microgravity could be due 

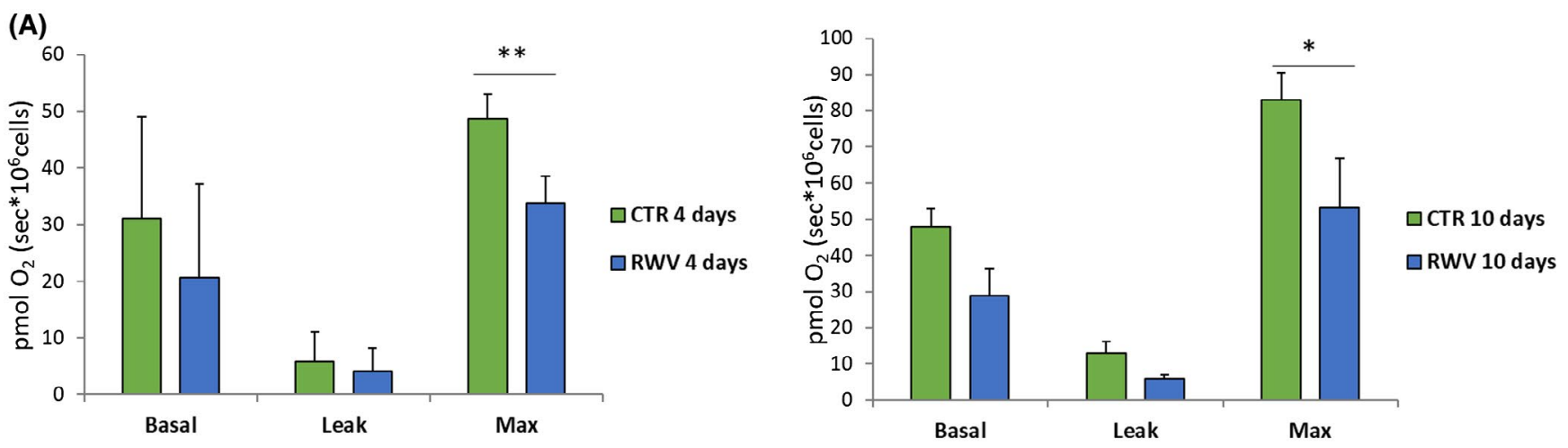

(B)
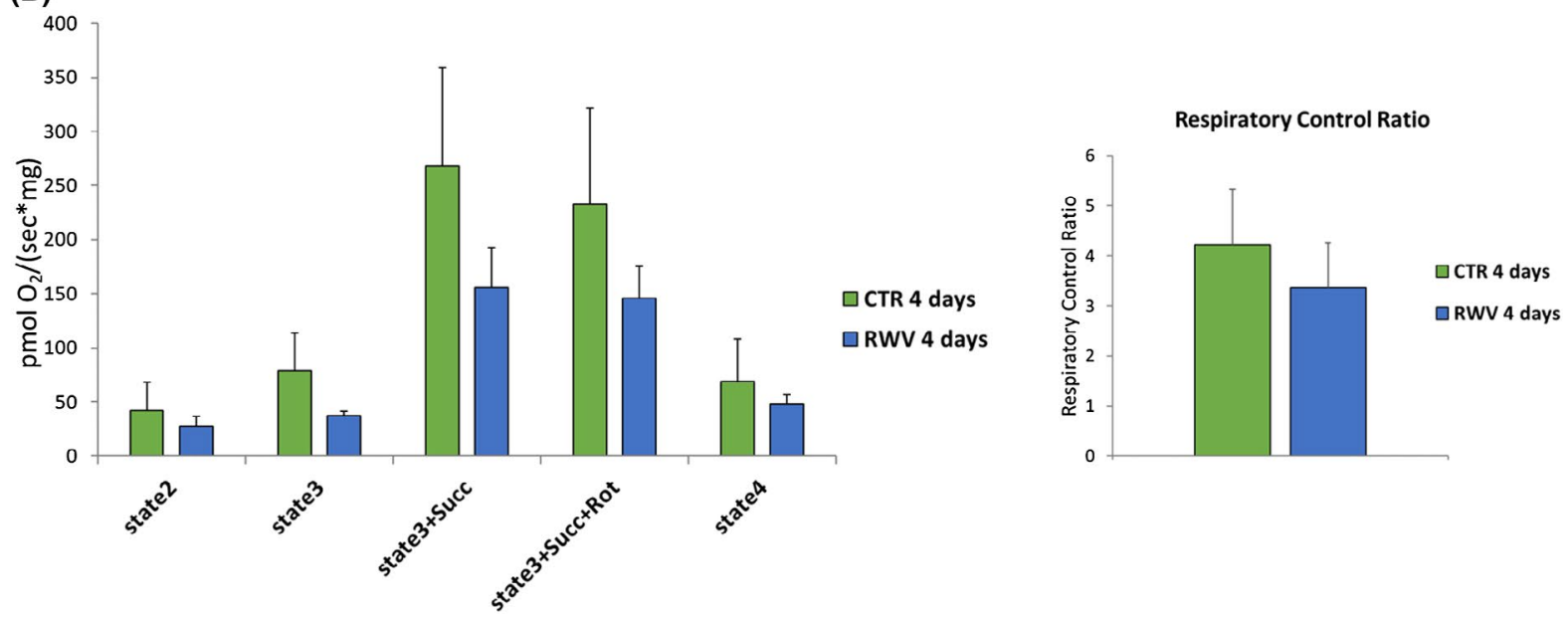

F I G U RE 3 High-resolution respirometry on HUVEC and on isolated mitochondria after exposure to simulated microgravity. HUVEC were cultured in $1 \mathrm{~g}$ condition (CTR) or in the RWV for 4 and $10 \mathrm{~d}$. A, High-resolution respirometry was performed on $1 \times 10^{6}$ cells and basal, leak, and maximal respiration were measured as described. B, Mitochondria were extracted from HUVEC grown for $4 \mathrm{~d}$ in $1 \mathrm{~g}$ condition (CTR) or in the RWV and oxygen consumption was measured on a purified fraction of mitochondria $(150 \mu \mathrm{g})$. The efficiency of the different complexes of the respiratory chain was measured. Glutamate $(10 \mathrm{mM})$ and malate $(2 \mathrm{mM})$ were used to measure complex I respiration (state2) and ADP $(2.5 \mathrm{mM})$ to reveal its maximal oxidative phosphorylation capacity (state3). Then, complexes I and II together were tested using succinate (10 mM) (state $3+$ Succ) and rotenone $(0.5 \mu \mathrm{M})$ was used to inhibit complex I in order to study complex II (state3+Succ+Rot). Oligomycin $(2 \mu \mathrm{g} / \mathrm{mL})$, an inhibitor of the ATP synthase, was used to measure proton leakage (state4). The respiratory control ratio was calculated as the ratio between state3 and state 4 and used as a general indicator of mitochondrial function. The experiments were repeated three times \pm standard deviation

to an acceleration of the autophagic flow and, specifically, to mitophagy. Initially, on the basis of studies indicating the activation of autophagy in HUVEC in simulated microgravity, ${ }^{29-32}$ we evaluated two markers of autophagy by western blot, ie, microtubule-associated proteins $1 \mathrm{~A} / 1 \mathrm{~B}$ light chain 3B (LC3 B) and Sequestosome 1 (p62). LC3 B is involved in the formation of autophagosomal vacuoles, while p62 is expressed with the cargo within the vesicle. Since LC3 B is cleaved when autophagy occurs, we measured the ratio between the cleaved (LC3 B-II) and total (LC3 B-I) forms of the protein. Western Blot revealed no significant modulation of these proteins in HUVEC exposed for $4 \mathrm{~d}$ to simulated microgravity vs their controls (Figure 4A). We hypothesized that autophagy in simulated microgravity was fast to the point that the autophagosomes were very rapidly degraded, preventing the possibility of detecting an increase of LC3 B-II or p62 changes. Therefore, on the basis of previous studies, ${ }^{33}$ we decided to treat the cells with CQ, an inhibitor of autophagy that blocks the binding of autophagosomes to lysosomes by altering the acidic environment of the lysosomes. ${ }^{33}$ This means that $\mathrm{CQ}$ allows the accumulation of autophagosomes and facilitates the detection of both LC3 B-II and p62 even in the case of very fast autophagic flow. HUVEC were cultured for 4 and $10 \mathrm{~d}$ in $1 \mathrm{~g}$ condition and in the RWV. One hour before ending the experiment, CQ $(40 \mu \mathrm{M})$ was added to culture media. Then, the cells were lysed and evaluated for markers of autophagy by western blot. Figure 4B reveals that HUVEC cultured in the RWV significantly upregulated LC3 B-II and p62, suggesting that the decreased content of mitochondria in simulated microgravity could be due to increased autophagy. Of note, we also detected higher amounts of the marker of mitophagy BNIP3, which targets mitochondria to autophagosomes by interacting directly with LC $3,{ }^{34}$ in HUVEC cultured in the RWV for 4 and $10 \mathrm{~d}$ vs their controls (Figure 4B). 
(A) 4 days

CTR RWV
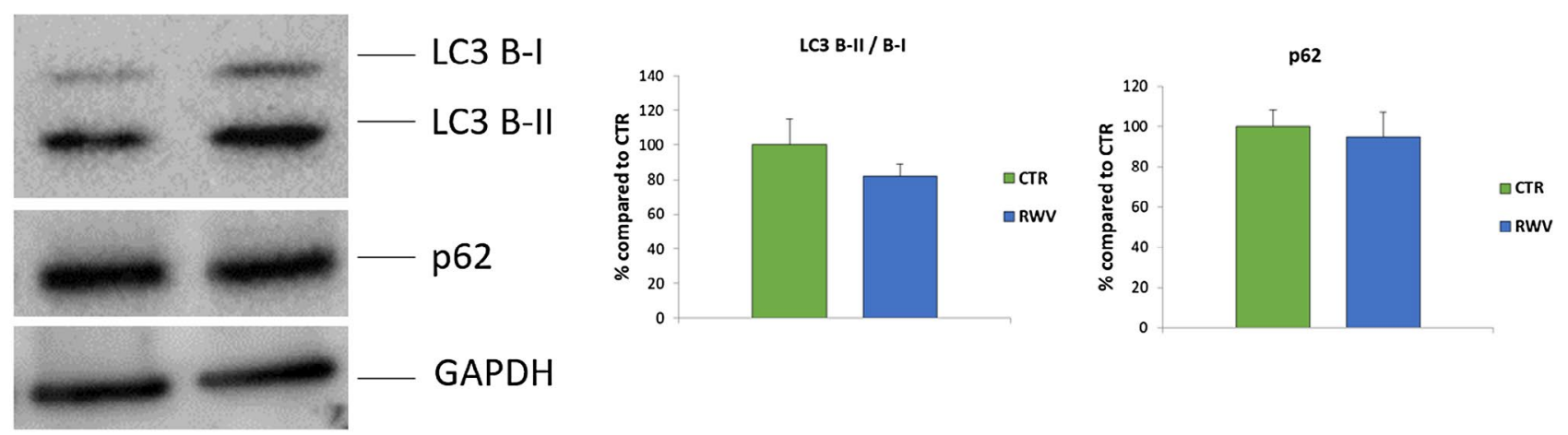

(B)

CQ
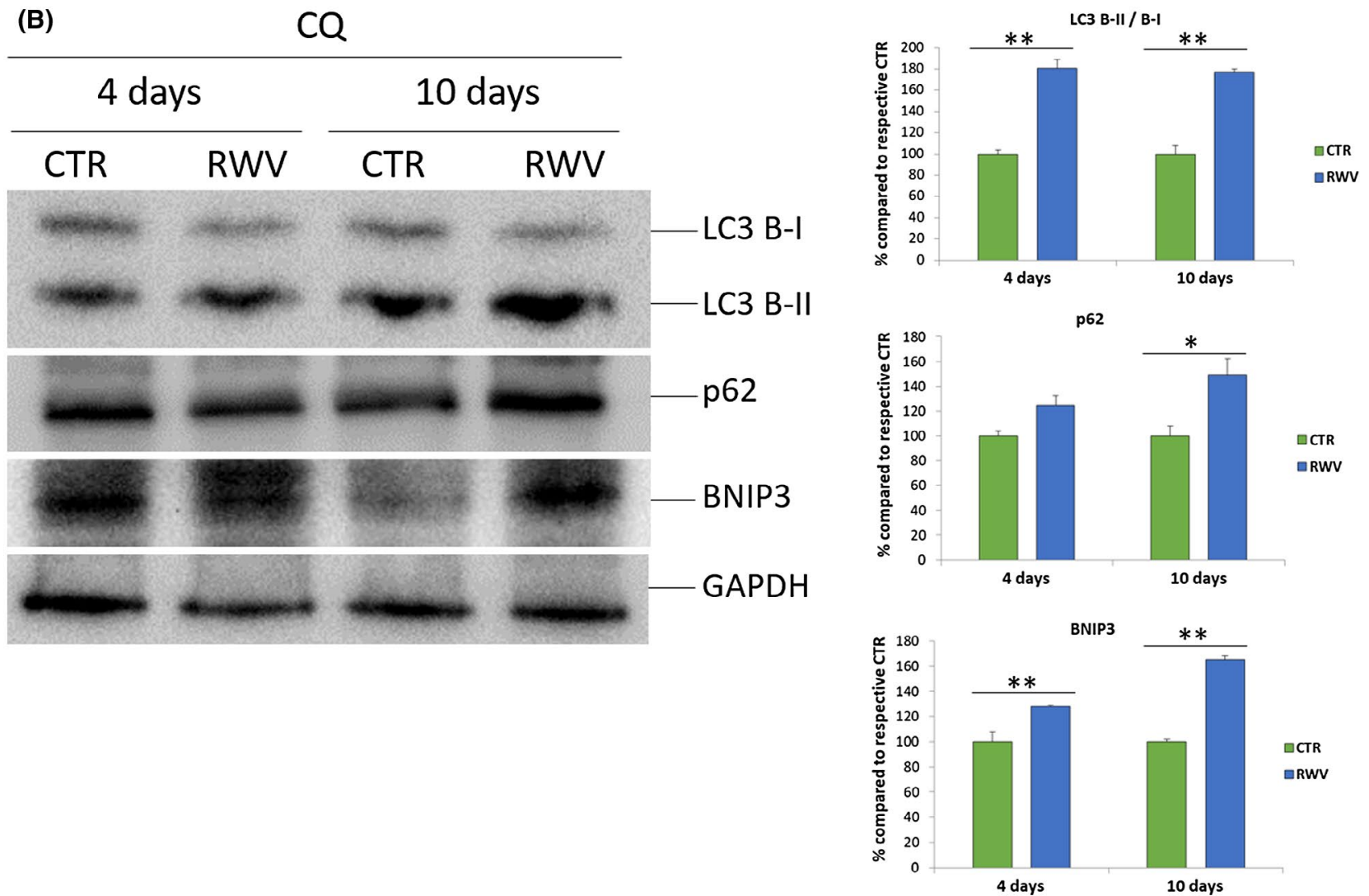

F I G U R E 4 The induction of autophagy in HUVEC in simulated microgravity. Markers of autophagy were investigated in HUVEC in 1g condition (CTR) or in the RWV. A, HUVEC were grown in 1g condition (CTR) or in the RWV for $4 \mathrm{~d}$. Western blot for two markers of autophagy, ie, LC3 B and p62, was performed. B, HUVEC were cultured 1g condition (CTR) or in the RWV for 4 and $10 \mathrm{~d}$. One hour before cell lysis, the cells were treated with chloroquine. Western blot was performed. GAPDH was used as a marker of loading. Representative blots (left) and densitometry obtained by ImageJ (right) are shown

\section{4 | The role of autophagy in modulating mitochondrial content in HUVEC exposed to simulated microgravity}

To link autophagy to the decrease of mitochondrial content in simulated microgravity, we inhibited autophagy/mitophagy using $\mathrm{CQ} .{ }^{25}$ After performing dose- and time-dependent cell viability experiments, $40 \mu \mathrm{M}$ emerged as the ideal concentration of CQ to inhibit autophagy/mitophagy without affecting HUVEC survival up to $4 \mathrm{~d}$ of treatment (data not shown). We cultured HUVEC in the RWV in the presence or in the absence of CQ for $4 \mathrm{~d}$. Immunofluorescence for LC3 B 
(A)

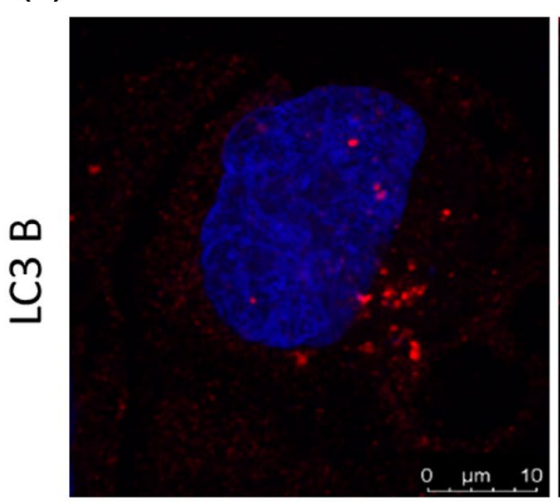

(B)

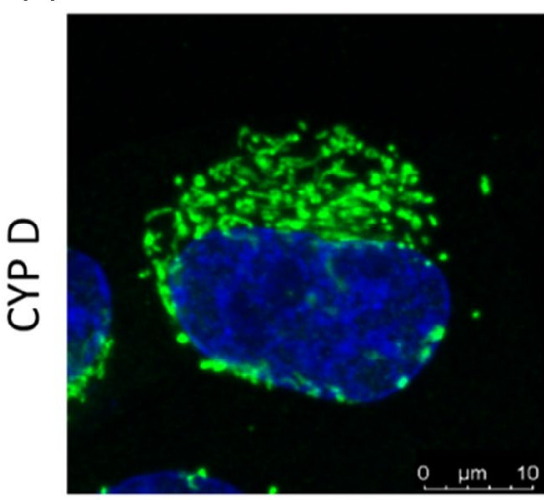

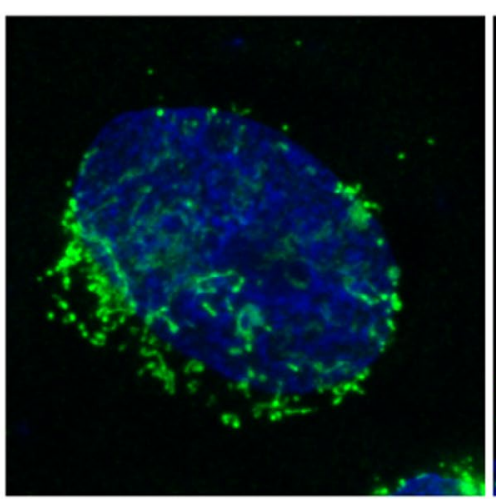

RWV

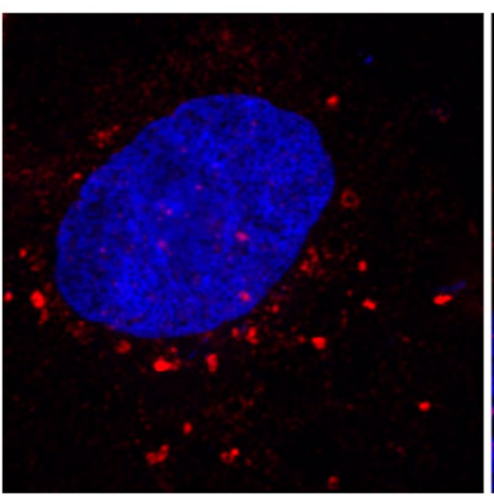

RWV

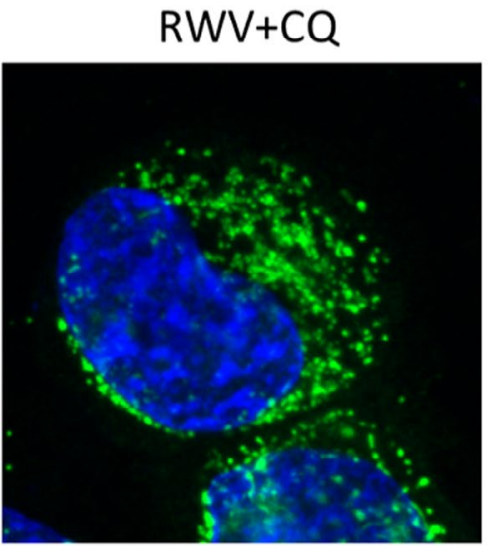

CTR

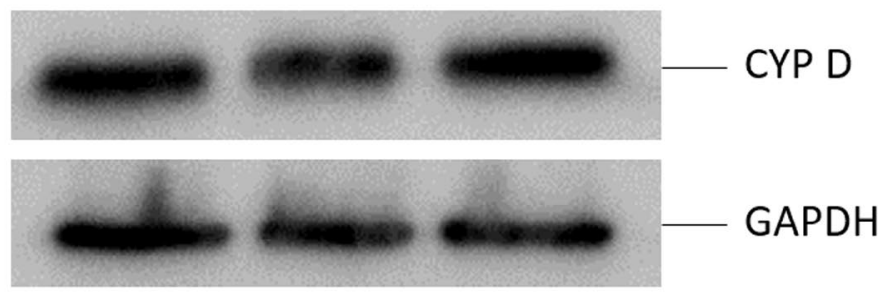

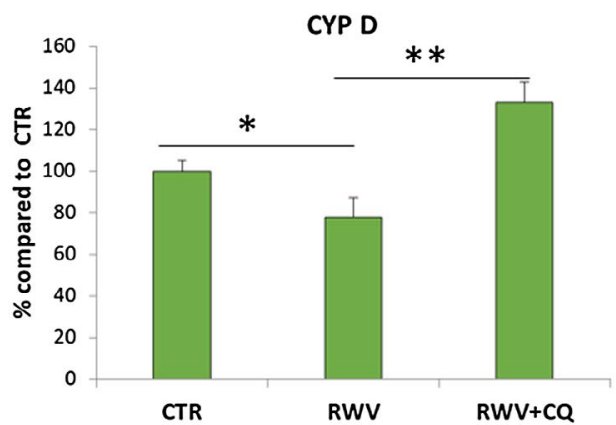

F I G U RE 5 Mitochondrial content in HUVEC exposed to simulated microgravity after inhibiting autophagy. HUVEC were cultured with (RWV+CQ) or without (CTR and RWV) chloroquine $(40 \mu \mathrm{M})$ for $4 \mathrm{~d}$. A, Immunofluorescence with antibodies against LC3 B was performed to show the inhibition of autophagy by chloroquine. Nuclei were stained by DAPI. B, Mitochondria were visualized utilizing antibodies against CYP D (upper panel) and Western blot was performed on cell lysates (lower panel). A representative blot is shown on the left. Densitometry was performed using Image $\mathrm{J}$ and is shown on the right

(Figure 5A) demonstrates that CQ actually inhibits the degradation of the autophagosomes. Then, immunofluorescence and western blot for the mitochondrial protein CYP D were performed on HUVEC grown in $1 \mathrm{~g}$ condition, in simulated microgravity (RWV) in the presence or in the absence of chloroquine (RWV+CQ) for $4 \mathrm{~d}$. When autophagy was blocked by CQ, HUVEC cultured in the RWV, and their controls had a similar mitochondrial content, thus demonstrating that autophagy is responsible for the degradation of mitochondria in simulated microgravity (Figure 5B).

\section{5 | The role of cytoskeletal disorganization in driving mitophagy and reducing mitochondrial content}

We then asked what triggers autophagy in HUVEC in the RWV. Since we have detected a mild and transient increase of ROS in simulated microgravity, ${ }^{15}$ we performed experiments in the presence of NAC $(1 \mathrm{mM}) .{ }^{15}$ HUVEC were cultured in the RWV or under $1 \mathrm{~g}$ condition in the presence or not of NAC for 4 hours. Some cells were removed from the RWV after 2 and 4 hours to measure the amounts of ROS 

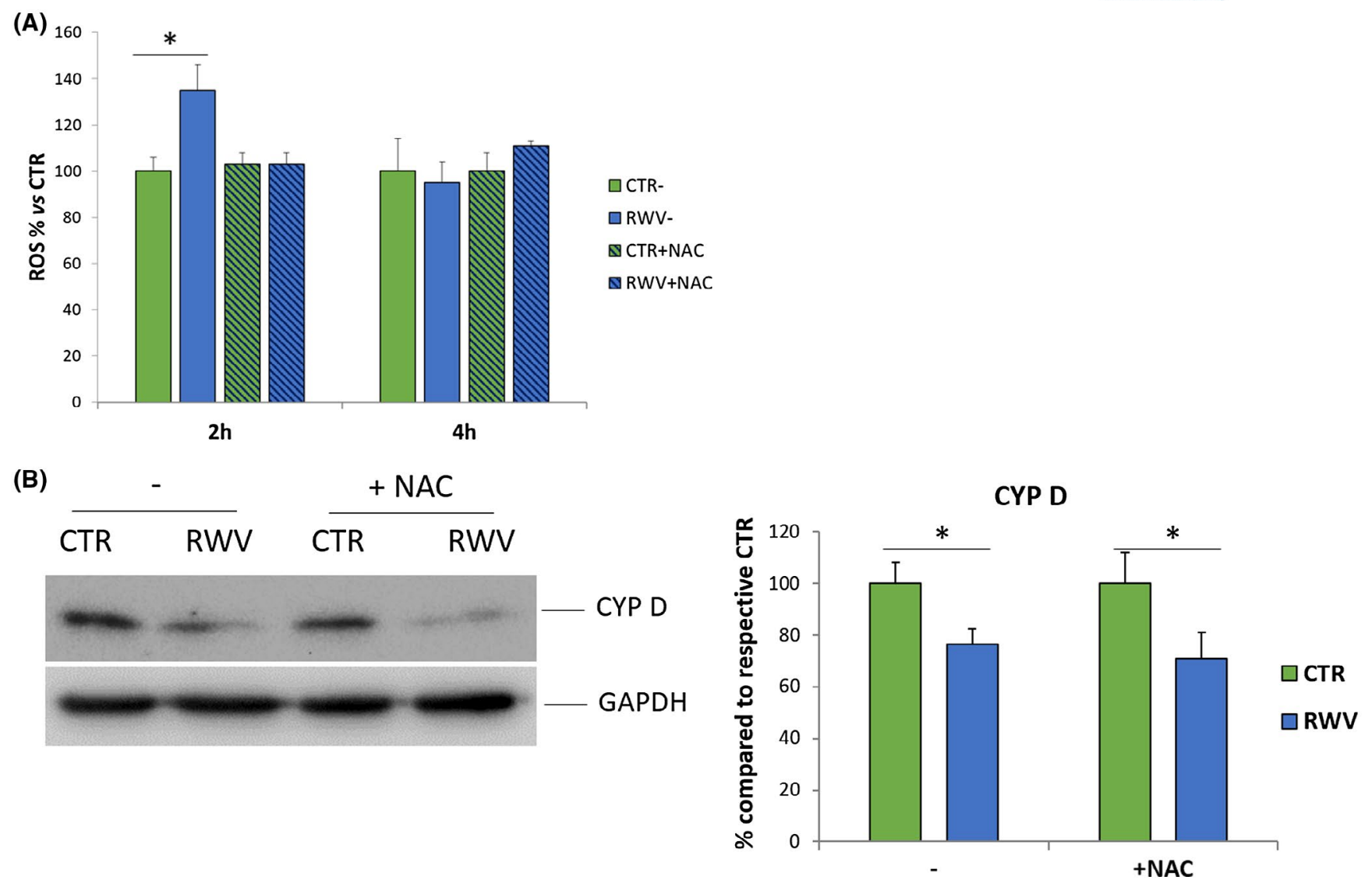

F I G URE 6 Mitochondrial content in HUVEC exposed to the anti-oxidant NAC. HUVEC were treated with NAC (1 mM) for 4 hours and then maintained in the RWV for $10 \mathrm{~d}$. A, After 2 and 4 hours of culture in the RWV, ROS were measured using DCFH as described. B, At the end of the experiment, western blot was performed on cell lysates using antibodies against CYP D. GAPDH was used as a control of loading. Densitometry was performed by Image J (right)

using DCFH. Figure 6A shows that NAC prevented the accumulation of ROS in HUVEC cultured in the RWV for 2 hours. At the end of the experiment (day 10), no differences in mitochondrial mass were observed between HUVEC in the RWV with or without NAC as shown by western blot with antibodies against CYP D (Figure 6B), thus ruling out a role of oxidant species in decreasing mitochondrial content.

We then anticipated that cytoskeletal disorganization, which occurs upon gravitational unloading, ${ }^{3,7,8,10}$ might be implicated in reducing mitochondria in simulated microgravity. As previously shown, ${ }^{3,7,8,10}$ while controls have well organized abundant stress fibers, HUVEC in the RWV for 96 hours show disassembly and disorganization of actin fibers (Figure 7A). Therefore, we mimicked cytoskeletal disruption in $1 \mathrm{~g}$ condition using Cytochalasin $\mathrm{D}$, a toxin that binds actin, and induces its depolymerization. We performed dose- and time-dependent experiments to test cell viability, and found that $0.5 \mu \mathrm{M}$ Cytochalasin D was not cytotoxic up to 96 hours (data not shown). HUVEC were then treated with $0.5 \mu \mathrm{M}$ Cytochalasin D for 24 hours. We observed massive disorganization of actin fibers associated with modifications of cell shape (Figure 7B), which closely recalls the results obtained in microgravity (Figure 8). ${ }^{3,7,8,10}$ Focusing on the mitochondria of Cytochalasin D-treated HUVEC, both immunofluorescence and western blot showed a decrease of CYP D (Figure 7B,C), indicating a reduced mitochondrial mass. Interestingly, Figure $7 \mathrm{C}$ also shows the upregulation of BNIP3. It is noteworthy that the concomitant exposure of HUVEC to cytochalasin D and simulated microgravity for 24 hours resulted in massive cell death (not shown).

We propose that cytoskeletal disruption occurring in simulated microgravity leads to increased mitophagy which results in a decreased content of mitochondria (Figure 8).

\section{4 | DISCUSSION}

The rise of human activities on the ISS and in expected longterm space missions has boosted novel studies to unravel biochemical and structural alterations occurring in cells exposed to microgravity. It is now clear that cells and tissues activate adaptive responses to cope with the absence of gravity, the only environmental factor which has remained constant since when life began on Earth. In particular, the endothelium, the inner lining of blood vessels, dynamically adapts to microgravity through the sequential intervention 
(A)

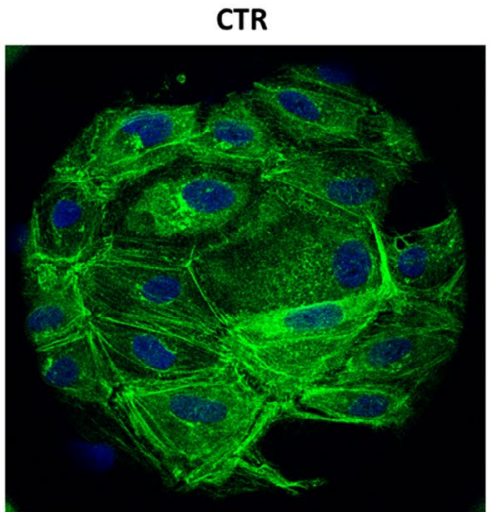

CTR

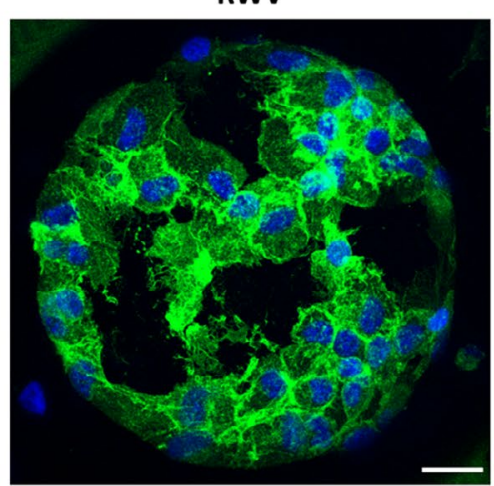

(C)
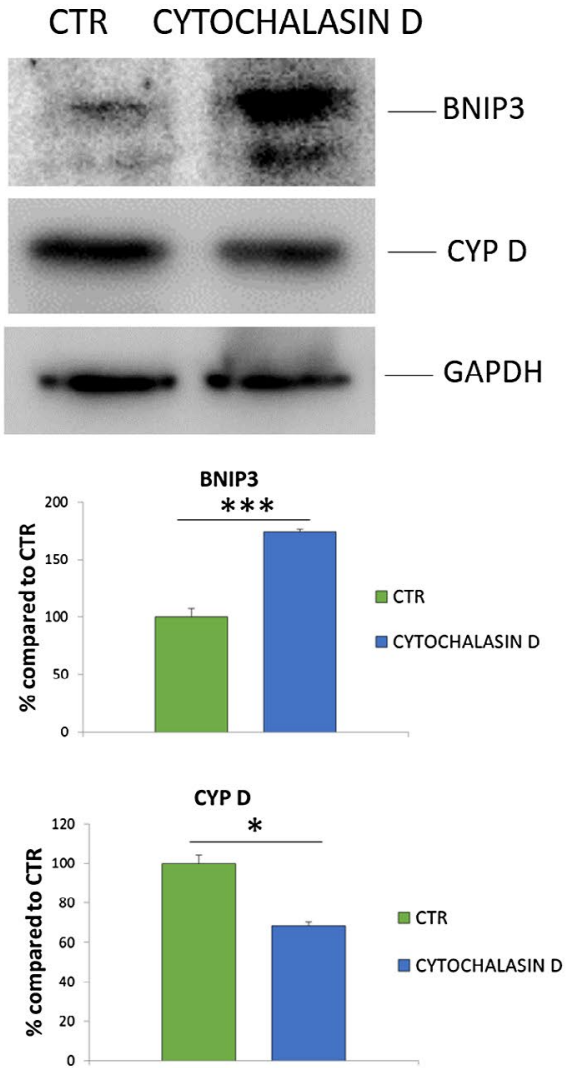

(B)
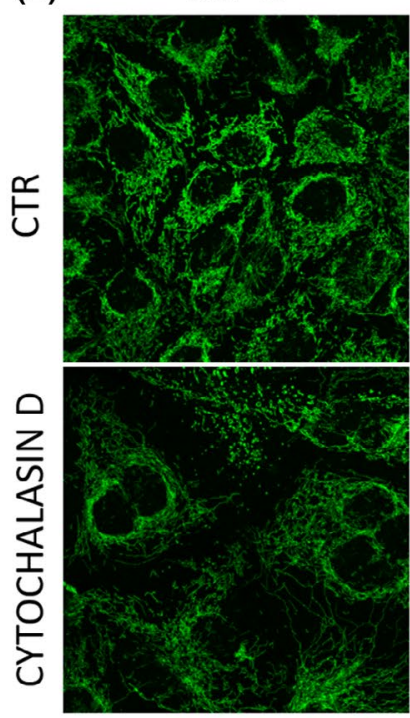

ACTIN

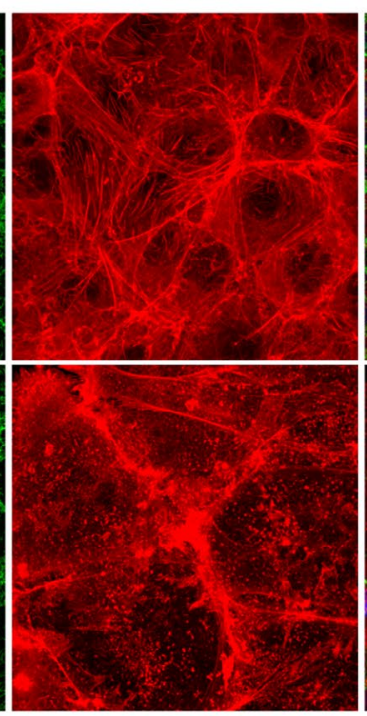

MERGE

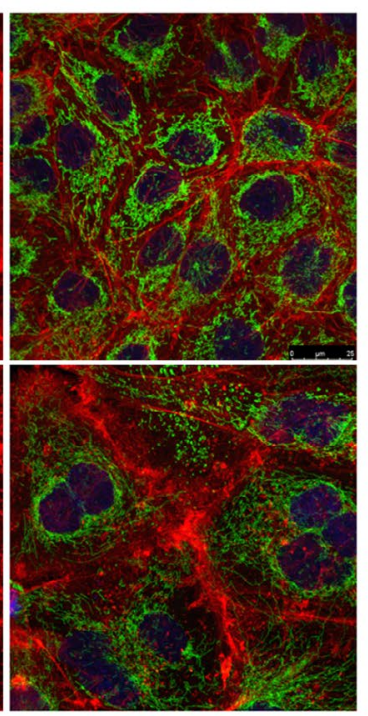

F I G U RE 7 Mitochondrial content and mitophagy in HUVEC exposed to Cytochalasin D. A, HUVEC were exposed to simulated microgravity for 96 hours. Cytoskeleton was visualized after staining with fluorescein-labeled phalloidin and nuclei with DAPI. B, HUVEC were treated or not with Cytochalasin D for 24 hours and stained with antibodies against CYP D (left) and with rhodamine-labeled phalloidin (middle). The merge is shown in the right panels. C, BNIP3 and CYP D were analyzed by western blot. GAPDH was used as a control of loading. The experiments were repeated at least three times. Representative blots (upper panel) and relative quantification (lower panel) obtained by ImageJ are shown

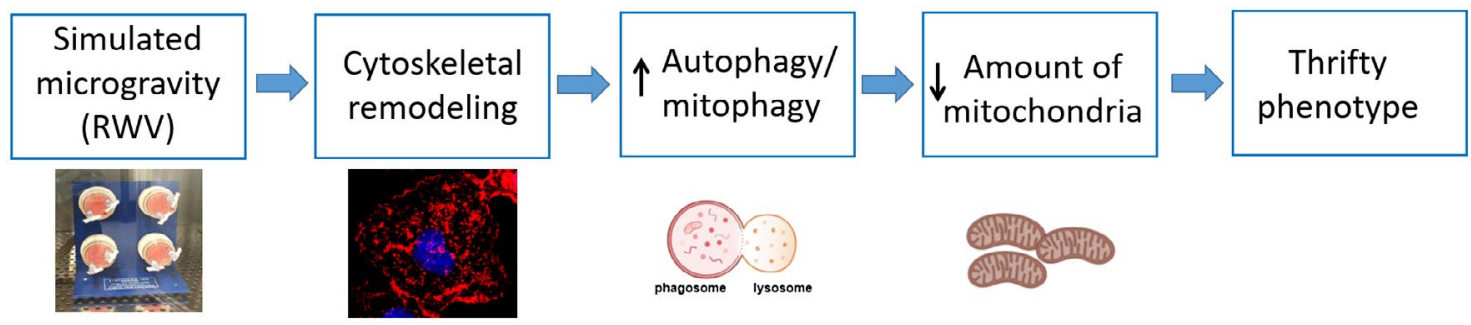

F I G U RE 8 A summary about the mechanisms leading to decreased mitochondrial mass in HUVEC cultured in simulated microgravity. We propose that cytoskeletal disruption triggers autophagy/mitophagy which reduces mitochondrial content and leads to the acquisition of a thrifty phenotype

of various stress proteins and acquires novel features that maintain cellular homeostasis. ${ }^{15}$ Also autophagy, a process which allows the cell to raze redundant and/or dysfunctional components, is considered a survival adaptive response, thus contributing to stress resistance. ${ }^{35}$ Accordingly, various types of stress--such as heat shock and mechanical cues-activate autophagy in various cell types. ${ }^{36,37}$ Therefore, it is not surprising that simulated microgravity induces 
autophagy. ${ }^{29-31}$ In HUVEC, clinorotation, a simulated model of microgravity, enhances autophagosome formation ${ }^{29}$ via downregulation of mTOR by a murine double minute $2 /$ p53 dependent mechanisms. ${ }^{30}$ In agreement with these results, we report that simulated microgravity generated by the RWV induces autophagy in HUVEC. Our novel finding is that increased autophagy results in mitochondria degradation, since blocking autophagy with CQ prevents mitochondrial loss. Interestingly, this event is reversible upon return to $1 \mathrm{~g}$ condition, a finding which suggests the adaptive nature of the phenomenon. In addition to increased amounts of LC3 B-II, also BNIP3, a mitochondrial stress sensor and a crucial player in mitophagy, ${ }^{34}$ is upregulated in HUVEC exposed to microgravity. Mitophagy has a housekeeping role in degrading damaged mitochondria and also entails the clearance of healthy mitochondria under stress. ${ }^{38}$

While $2 \mathrm{~d}$ of gravitational unloading activate autophagy of Hodgkin's lymphoma cells through the accumulation of ROS,${ }^{31}$ a modest increase of ROS was reported in HUVEC only after 2 hours in simulated microgravity. ${ }^{15}$ These results indicate that the time-response of HUVEC and lymphoma cells and their ability to counterbalance oxidative stress are very different. We hypothesize that HUVEC retain a higher anti-oxidant arsenal than lymphoma cells. Indeed, in HUVEC gravitational unloading upregulates several proteins that scavenge free radicals. ${ }^{15}$ In addition, because the antioxidant NAC does not prevent mitochondrial loss in HUVEC in microgravity, we propose that ROS are not implicated in promoting autophagy in these cells.

Another common feature of cells in microgravity is cytoskeletal disorganization and, notably, the cytoskeleton has been proposed as a sensor of changes of gravity. ${ }^{39}$ Since (a) cytoskeletal dynamics have an important role in autophagy, ${ }^{40}$ (b) mitochondria interact with the cytoskeletal components, ${ }^{21}$ (c) mitophagy is regulated by cytoskeletal components, ${ }^{41}$ and (d) microgravity rapidly and steadily disassembles HUVEC cytoskeleton, ${ }^{3,7,8,10}$ we anticipate that a link might exist between autophagy, mitochondria content, and cytoskeletal remodeling in our experimental model. Indeed, Cytochalasin D closely mimics microgravity-induced cytoskeletal disorganization and also reduces mitochondrial content through mitophagy. We conclude that the remodeling of the cytoskeleton in microgravity plays a role in activating mitophagy, therefore leading to reduced mitochondrial content.

In HUVEC in the RWV mitophagy culminates with the reduction of mitochondrial mass which is associated with a reduced consumption of oxygen. However, mitochondria are as efficient in HUVEC in simulated microgravity and their controls. Since endothelial mitochondria are biosynthetic factories rather than powerhouse, the decreased mitochondrial mass might constrain synthetic pathways as an adaptive response to reach a new balance that allows survival and maintains fundamental functions. We propose that HUVEC in simulated microgravity acquire a thrifty phenotype and are less prone to face an overload of substrates. A lower amount of mitochondria might also protect HUVEC from an overproduction of ROS, thus integrating the complex modulation of stress proteins aimed at counteracting the upregulation of the pro-oxidant protein TXNIP. ${ }^{15}$ More studies are required to understand whether this adaptive response grants the maintenance of all the complex functions exerted by EC as well as their resisteance to further stresses. It should also be recalled that EC are remarkably heterogeneous across the vascular tree in terms of structure, gene expression, and function. ${ }^{42}$ Accordingly, we have shown significant differences in the behavior of HUVEC vs human dermal microvascular EC. ${ }^{43}$ It will therefore be relevant to extend our studies to different types of microvascular EC.

Mitochondria are emerging as relevant targets of microgravity. Indeed, mitochondrial mass was reduced also in Hodgkin's lymphoma cells cultured in a clinostat. ${ }^{31}$ In osteoblasts cultured in simulated microgravity using the Random Positioning Machine mitochondrial functions were impaired, ${ }^{44}$ with important metabolic consequences, but no quantification of mitochondrial mass was performed. On the contrary, rat cardiomyocytes cultured in the RWV upregulated mitochondrial proteins to maintain the energetics of the cells at the expense of protein synthesis, ${ }^{45}$ and oligodendrocytes, the myelin-forming cells in the central nervous system, increased mitochondrial respiration in simulated microgravity, suggesting an increase in Kreb's cycle flux. ${ }^{46}$ The aforementioned differences reported in various cell types might be due to the fact that mitochondria exhibit tissue-specific characteristics, including their number and their capacity of ATP synthesis. ${ }^{47}$ Therefore, it is feasible to propose that, while ECs, which are quiescent in normal conditions, can reduce their mitochondria without detrimental effects on their viability, other highly differentiated cells optimize their energetics to meet the challenge generated by gravitational unloading. If the scenario is complex in in vitro systems, it becomes even more intricate in humans. A significant decrease in the mtDNA/nDNA was described in hair samples from ten astronauts after 6 mo on the ISS. ${ }^{48}$ In contrast, within the "NASA Twin Study," higher levels of mtDNA were revealed in peripheral blood mononuclear cells after $1 \mathrm{y}$ in space. ${ }^{2}$ Space is a harsh extreme environment where astronauts are exposed to stresses other than microgravity, such as cosmic radiations, tight work schedule, isolation, etc. It is therefore complicated to interpret human results, which should be read also in the light of individual diversity. It is even harder to compare them with data obtained in vitro.

In conclusion, we propose that microgravity-induced cytoskeletal disorganization triggers the activation of autophagy/mitophagy, thus leading to mitochondrial loss. These events might be relevant to deal with the novel metabolic demands generated in microgravity and to reach a 
novel balance that maintains endothelial survival and fundamental function.

\section{ACKNOWLEDGMENTS}

This work was supported, in part, by the Italian Space Agency (ENDOSTEO project).

\section{CONFLICT OF INTEREST}

No potential conflict of interest was reported by the authors.

\section{AUTHOR CONTRIBUTIONS}

L. Locatelli performed most of the experiments; A. Cazzaniga helped with culture in the RWV; C. De Palma conducted respirometry; S. Castiglioni and J.A.M. Maier designed research; J.A.M. Maier wrote the manuscript; and all authors analyzed data.

\section{REFERENCES}

1. White RJ, Averner M. Humans in space. Nature. 2001;409 (6823):1115-1118.

2. Garrett-Bakelman FE, Darshi M, Green SJ, et al. The NASA Twins Study: a multidimensional analysis of a year-long human spaceflight. Science. 2019;364(6436):eaau8650.

3. Maier JA, Cialdai F, Monici M, Morbidelli L. The impact of microgravity and hypergravity on endothelial cells. BioMed Res Int. 2015;2015:434803

4. Cahill PA, Redmond EM. Vascular endothelium-gatekeeper of vessel health. Atherosclerosis. 2016;248:97-109.

5. Michiels C. Endothelial cell functions. $J$ Cell Physiol. 2003;196(3):430-443.

6. García-Cardeña G, Gimbrone MA, Jr. Biomechanical modulation of endothelial phenotype: implications for health and disease. Handb Exp Pharmacol. 2006;(176 Pt 2):79-95.

7. Carlsson SI, Bertilaccio MT, BallabioE, Maier JA. Endothelial stress by gravitational unloading: effects on cell growth and cytoskeletal organization. Biochim Biophys Acta. 2003;1642(3):173-179.

8. Kapitonova MY, Muid S, Froemming GR, et al. Real space flight travel is associated with ultrastructural changes, cytoskeletal disruption and premature senescence of HUVEC. Malays $J$ Pathol. 2012;34(2):103-113.

9. Kapitonova MY, Kuznetsov SL, Froemming GR, et al. Effects of space mission factors on the morphology and function of endothelial cells. Bull Exp Biol Med. 2013;154(6):796-801.

10. Versari S, Villa A, Bradamante S, Maier JAM. Alterations of the actin cytoskeleton and increased nitric oxide synthesis are common features in human primary endothelial cell response to changes in gravity. Biochim et Biophys Acta. 2007;1773(11):1645-1652.

11. Versari S, Longinotti G, Barenghi L, Maier JAM, Bradamante S. The challenging environment on board the International Space Station affects endothelial cell function by triggering oxidative stress through thioredoxin interacting protein overexpression: the ESA-SPHINX experiment. FASEB J. 2013;27(11):4466-4475.

12. Grenon SM, Jeanne M, Aguado-Zuniga J, Conte MS, HughesFulford M. Effects of gravitational mechanical unloading in endothelial cells: association between caveolins, inflammation and adhesion molecules. Sci Rep. 2013;3:1494.
13. Griffoni C, Di Molfetta S, Fantozzi L, et al. Modification of proteins secreted by endothelial cells duringmodeled lowgravity exposure. J Cell Biochem. 2011;112(1):265-272.

14. Janmaleki M, Pachenari M, Seyedpour SM, Shahghadami R, Sanati-Nezhad A. Impact of simulated microgravity on cytoskeleton and viscoelastic properties of endothelial cell. Sci Rep. 2016;6:32418

15. Cazzaniga A, Locatelli L, Castiglioni S, Maier JAM. The dynamic adaptation of primary human endothelial cells to simulated microgravity. FASEB J. 2019;33(5):5957-5966.

16. De Bock K, Georgiadou M, Carmeliet P. Role of endothelial cell metabolism in vessel sprouting. Cell Metab. 2013;18(5):634-647.

17. Helmlinger G, Endo M, Ferrara N, Hlatky L, Jain RK. Formation of endothelial cell networks. Nature. 2000;405(6783):139-141.

18. Rohlenova K, Veys K, Miranda-Santos I, De Bock K, Carmeliet P. Endothelial cell metabolism in health and disease. Trends Cell Biol. 2018;28(3):224-236.

19. Caja S, Enríquez JA. Mitochondria in endothelial cells: sensors and integrators of environmental cues. Redox Biol. 2017;12:821-827.

20. Kluge MA, Fetterman JL, Vita JA. Mitochondria and endothelial function. Circ Res. 2013;112(8):1171-1188.

21. Moore AS, Holzbaur ELF. Mitochondrial-cytoskeletal interactions: dynamic associations that facilitate network function and remodeling. Curr Opin Physiol. 2018;3:94-100.

22. Grimm D, Wehland M, Pietsch J, et al. Growing tissues in real and simulated microgravity: new methods for tissue engineering. Tissue Eng Part B Rev. 2014;20(6):555-566.

23. Martinez EM, Yoshida MC, Candelario TL, Hughes-Fulford M. Spaceflight and simulated microgravity cause a significant reduction of key gene expression in early T-cell activation. Am J Physiol Regul Integr Comp Physiol. 2015;308(6):R480-R488.

24. Cotrupi S, Ranzani D, Maier JAM. Impact of modeled microgravity on microvascular endothelial cells. Biochim Biophys Acta. 2005;1746(2):163-168.

25. Georgakopoulos ND, Wells G, Campanella M. The pharmacological regulation of cellular mitophagy. Nat Chem Biol. 2017;13(2):136-146.

26. Ezeriņa D, Takano Y, Hanaoka K, Urano Y, Dick TP. N-acetyl cysteine functions as a fast-acting antioxidant by triggering intracellular H(2)S and sulfane sulfur production. Cell Chem Biol. 2018;25(4):447-459.e4.

27. Castiglioni S, Cazzaniga A, Trapani V, et al. Magnesium homeostasis in colon carcinoma LoVo cells sensitive or resistant to doxorubicin. Sci Rep. 2015;5:16538.

28. Elrod JW, Molkentin JD. Physiologic functions of cyclophilin $\mathrm{D}$ and the mitochondrial permeability transition pore. Circ J. 2013;77(5):1111-1122.

29. Wang YC, Lu DY, Shi F, et al. Clinorotation enhances autophagy in vascular endothelial cells. Biochem Cell Biol. 2013;91(5):309-314.

30. Li CF, Sun JX, Gao Y, et al. Clinorotation-induced autophagy via HDM2-p53-mTOR pathway enhances cell migration in vascular endothelial cells. Cell Death Dis. 2018;9(2):147.

31. Jeong AJ, Kim YJ, Lim MH, et al. Microgravity induces autophagy via mitochondrial dysfunction in human Hodgkin's lymphoma cells. Sci Rep. 2018;8(1):14646.

32. Li CF, Pan YK, Gao Y, Shi F, Wang YC, Sun XQ. Autophagy protects HUVECs against ER stress-mediated apoptosis under simulated microgravity. Apoptosis. 2019;24(9-10):812-825. 
33. Klionsky DJ, Abdelmohsen K, Abe A, et al. Guidelines for the use and interpretation of assays for monitoring autophagy. Autophagy. 2016;12(1):1-222.

34. Rikka S, Quinsay MN, Thomas RL, et al. BNIP3 impairs mitochondrial bioenergetics and stimulates mitochondrial turnover. Cell Death Differ. 2011;18(4):721-731.

35. Murrow L, Debnath J. Autophagy as a stress-response and quality-control mechanism: implications for cell injury and human disease. Annu Rev Pathol. 2013;8:105-137.

36. Dokladny K, Myers OB, Moseley PL. Heat shock response and autophagy-cooperation and control. Autophagy. 2015;11(2):200-213.

37. King JS. Mechanical stress meets autophagy: potential implications for physiology and pathology. Trends Mol Med. 2012;18 (10):583-588.

38. Egan DF, Shackelford DB, Mihaylova MM, et al. Phosphorylation of ULK1 (hATG1) by AMP-activated protein kinase connects energy sensing to mitophagy. Science. 2011;331(6016):456-461.

39. Vorselen D, Roos WH, MacKintosh FC, Wuite GJ, van Loon JJ. The role of the cytoskeleton in sensing changes in gravity by nonspecialized cells. FASEB J. 2014;28(2):536-547.

40. Kast DJ, Dominguez R. The cytoskeleton-autophagy connection. Curr Biol. 2017;27(8):R318-R326.

41. Strzyz P. Autophagy: mitochondria encaged. Nat Rev Mol Cell Biol. 2018;19(4):212.

42. Aird WC. Endothelial cell heterogeneity. Cold Spring Harb Perspect Med. 2012;2(1):a006429.
43. Mariotti M, Maier JA. Gravitational unloading induces an anti-angiogenic phenotype in human microvascular endothelial cells. J Cell Biochem. 2008;104(1):129-135.

44. Michaletti A, Gioia M, Tarantino U, Zolla L. Effects of microgravity on osteoblast mitochondria: a proteomic and metabolomics profile. Sci Rep. 2017;7(1):15376.

45. Feger BJ, Thompson JW, Dubois LG, et al. Microgravity induces proteomics changes involved in endoplasmic reticulum stress and mitochondrial protection. Sci Rep. 2016;6:34091.

46. Espinosa-Jeffrey A, Nguyen K, Kumar S, et al. Simulated microgravity enhances oligodendrocyte mitochondrial function and lipid metabolism. J Neurosci Res. 2016;94(12):1434-1450.

47. Kunz WS. Different metabolic properties of mitochondrial oxidative phosphorylation in different cell types-important implications for mitochondrial cytopathies. Exp Physiol. 2003;88(1):149-154.

48. Indo HP, Majima HJ, Terada M, et al. Changes in mitochondrial homeostasis and redox status in astronauts following long stays in space. Sci Rep. 2016;6:39015.

How to cite this article: Locatelli L, Cazzaniga A, De Palma C, Castiglioni S, Maier JAM. Mitophagy contributes to endothelial adaptation to simulated microgravity. The FASEB Journal. 2020;34:18331845. https://doi.org/10.1096/fj.201901785RRR 